\title{
Characteristics and limitations of GPS L1 observations from submerged antennas
}

\author{
Theoretical investigation in snow, ice, and freshwater and practical observations within a \\ freshwater layer
}

Ladina Steiner $^{1} \cdot$ Michael Meindl $^{1} \cdot$ Alain Geiger $^{1}$

Received: 15 May 2017 / Accepted: 25 April 2018 / Published online: 11 May 2018

(c) The Author(s) 2018

\begin{abstract}
Observations from a submerged GNSS antenna underneath a snowpack need to be analyzed to investigate its potential for snowpack characterization. The magnitude of the main interaction processes involved in the GPS L1 signal propagation through different layers of snow, ice, or freshwater is examined theoretically in the present paper. For this purpose, the GPS signal penetration depth, attenuation, reflection, refraction as well as the excess path length are theoretically investigated. Liquid water exerts the largest influence on GPS signal propagation through a snowpack. An experiment is thus set up with a submerged geodetic GPS antenna to investigate the influence of liquid water on the GPS observations. The experimental results correspond well with theory and show that the GPS signal penetrates the liquid water up to three centimeters. The error in the height component due to the signal propagation delay in water can be corrected with a newly derived model. The water level above the submerged antenna could also be estimated.
\end{abstract}

Keywords GNSS signal propagation in snow, ice, water $\cdot$ Signal strength attenuation $\cdot$ Water depth $\cdot$ Sub-snow

\section{Introduction}

Extensive amount of water stored in snow covers has a strong impact on flood development during snow melting periods. Early assessment of the snow water equivalent or wetness 1 in mountain environments enhances early warning and thus prevention of major impacts. Sub-snow GNSS techniques are lately suggested to determine liquid water content (Koch et al. 2014) or considered for avalanche rescue (Schleppe and Lachapelle 2008; Olmedo et al. 2012). Schleppe and Lachapelle (2008) analyzed experimentally the GPS tracking performance under avalanche deposited snow at two test sites

\footnotetext{
1 Wetness or liquid water content is defined as the amount of water within the snow that is in the liquid phase. Snow water equivalent is the depth of water that would result if the mass of snow melted completely (Fierz et al. 2009).
}

Ladina Steiner

ladinasteiner@ethz.ch

1 Institute of Geodesy and Photogrammetry, ETH Zurich, Robert-Gnehm-Weg 15, 8093 Zurich, Switzerland in Canada. The potential of a GPS-based rescue system for victims buried under avalanches was investigated by Olmedo et al. (2012) in the framework of the SICRA project. Koch et al. (2014) estimated the liquid water content (wetness) continuously based on GPS L1 observations for a seasonal snowpack in the Swiss Alps.

Furthermore, GNSS stations above a snowpack are used for snow depth estimation based on the interference of direct and reflected GNSS signals (GNSS-reflectometry). Thereby, the snow depth is estimated using GPS L1 C/A or L2c signalto-noise ratio (e. g. Larson et al. 2009; Boniface et al. 2015). Ozeki and Heki (2012) estimated snow depth from permanent GPS networks based on a geometry-free linear combination from GPS phase observations. Cardellach et al. (2012) investigated the potential to remotely sense sub-surface snow structures in dry snow areas using bi-statically reflected GNSS signals.

However, GNSS observations for applications within a snowpack still need to be further investigated. How are GNSS observations influenced when placing a GNSS antenna in an unusual environment, e.g., snow, water, or ice instead of air? 
Limitations in signal penetration depth and satellite tracking performance, as well as characteristics in signal attenuation and the coordinate solutions quality from a submerged GNSS antenna are analyzed and quantified in the present paper. Liquid water is assumed to be the limiting factor within a snowpack as it has the highest influence on the GNSS observations. Therefore, the characteristics of GPS observations within a freshwater layer are analyzed experimentally. This study serves as a preparatory work to investigate snowpack properties based on submerged GPS antennas within a snowpack.

Section 2 presents the theoretical background of the main interaction processes for a signal propagating through a medium. Four typical snow wetness classes [dry, moist, wet, very wet snow, as defined in Table 1 according to Fierz et al. (2009)], ice, and freshwater at $0{ }^{\circ} \mathrm{C}$ are explicitly examined. The highest influence on the GPS observations is thereby assumed for the presence of liquid water. This assumption is experimentally analyzed in Sect. 3 using a geodetic GPS antenna (Leica AS10) submerged into water. A model is developed to correct for the excess path length due to the delaying medium. It can be used to improve the coordinates estimation if the water level above the submerged antenna is known or to estimate the water depth if the coordinates are known. Section 4 concludes the paper.

\section{GNSS signal propagation in snow, ice, and water}

An overview of the general geometry when an electromagnetic wave propagates into a single layer of snow, ice, or water with depth $d$ is visible in Fig. 1. A GPS reference antenna is located in a short distance outside the medium and is not affected by the medium (Fig. 1, noted as Ant ref). Additionally, a submerged GPS antenna (noted as $\mathrm{Ant}_{\mathrm{m}}$ ) is located on the ground below a layer of the respective medium. Thereby, the GNSS signal is highly influenced while propagating through the medium until it reaches the submerged antenna. The power of the wave at different locations along its path is indicated by $P$. The incident zenith angle of the wave is referred as $v_{\mathrm{a}}$ and the corresponding refractive angle after refraction at the air/medium boundary as $v_{\mathrm{m}}$. The refractive indices of air and the medium are defined by $n_{\mathrm{a}}$ and $n_{\mathrm{m}}$, respectively:

$n_{\mathrm{a}}<n_{\mathrm{m}} \quad$ or $\quad c_{\mathrm{a}}>c_{\mathrm{m}}$

where $c_{\mathrm{a}}$ and $c_{\mathrm{m}}$ refer to the propagation velocities in air and the medium and depend on the vacuum light velocity $c_{0}=299^{\prime} 792^{\prime} 458 \frac{\mathrm{m}}{\mathrm{s}}$ (Woodhouse 2005):

$c_{\mathrm{a}}=\frac{c_{0}}{n_{\mathrm{a}}} \quad$ and $\quad c_{\mathrm{m}}=\frac{c_{0}}{n_{\mathrm{m}}}$

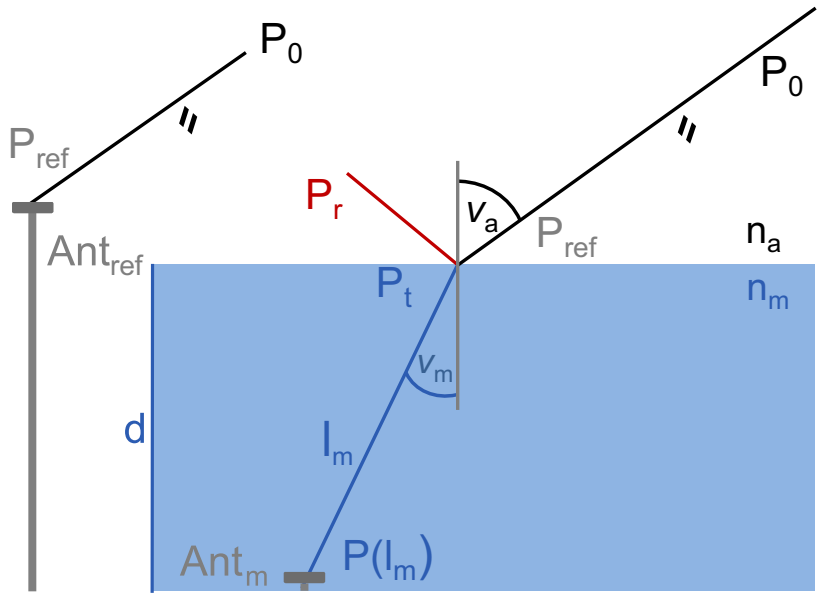

Fig. 1 Overview of interaction processes at the air/medium boundary. Modified according to Koch et al. (2014)

The power $P_{0}$ of the electromagnetic wave sent from a satellite undergoes varying losses in the atmosphere before reaching a single layer of snow, ice, or water at the Earth's surface. The incident power $P_{\text {ref }}$ at the air/medium boundary is equally affected by the atmospheric losses as the power received at the short baseline reference station (Fig. 1).

A part $P_{\mathrm{r}}$ of the signal power is reflected at the air/medium boundary. The transmitted signal power $P_{\mathrm{t}}$ into the medium is refracted at the boundary. The magnitude of reflection and refraction depends on the corresponding refractive index $n_{\mathrm{m}}$ or relative permittivity $\epsilon_{\mathrm{r}, \mathrm{m}}$ (Sect. 2.1) of the medium. While propagating through the present medium, the power of the signal is attenuated exponentially. The submerged antenna receives the remaining signal power $P\left(l_{\mathrm{m}}\right)$ related to the length of the signal path $l_{\mathrm{m}}$ in the medium. The main parameter quantifying these processes is the relative permittivity. These processes are thoroughly summarized by Koch et al. (2014).

The present paper assumes a single-layer model for simplification and neglects reflections from the bottom of the layer (ground reflections). Snow microstructure is negligible as the snow particles (around $1 \mathrm{~mm}$, Fierz et al. 2009) are much smaller than the GPS L1 wavelength of about $19 \mathrm{~cm}$. After introducing the relative permittivity in Sect. 2.1, the main effects reflection, refraction, and attenuation are described as well as their influence on the signal penetration depth. Finally, a model is derived in Sect. 2.6 to correct for the excess path length caused by the presence of a medium.

\subsection{Relative permittivity}

The relative permittivity $\epsilon_{\mathrm{r}}$ describes the frequency-dependent interaction of an electric field with a material relative to vacuum (Woodhouse 2005): 
$\epsilon_{\mathrm{r}}=\frac{\epsilon}{\epsilon_{0}}$,

Thereby, $\epsilon$ is the permittivity of the medium and $\epsilon_{0}$ the permittivity in vacuum. The dimensionless relative permittivity can be represented as a complex number:

$\epsilon_{\mathrm{r}}=\epsilon_{\mathrm{r}}^{\prime}+\mathrm{i} \epsilon_{\mathrm{r}}^{\prime \prime}$

The real part $\epsilon_{\mathrm{r}}^{\prime}$ of the complex relative permittivity describes the capacity which depends on snow wetness and dry snow density. The imaginary part $\epsilon_{\mathrm{r}}^{\prime \prime}$ (also called loss factor) is related to the energy absorption. The loss factor describes the transformation of electric field energy into inner energy of a medium and depends on frequency $f$.

Table 1 lists the relative permittivities for the different examined media at the GPS L1 wavelength. The relative permittivity for freshwater is according to Kaatze (1989); the values for ice are computed from the formulas given by Mätzler (1998). Please note that the loss factor for ice is only an approximation. Koh (1997), e.g., estimates a value of 0.003 for a frequency of $1.8 \mathrm{GHz}$ at $-2.5^{\circ} \mathrm{C}$. The author, however, recommends to use the values from Mätzler and Wegmüller (1987). The computed value of 0.0006 given in Table 1 conforms quite well to the referenced publication (Mätzler and Wegmüller 1987) and is therefore adopted in this section.

The real part of the relative permittivity for snow $\epsilon_{\mathrm{r}, \mathrm{s}}^{\prime}$ can be calculated from the three phase mixing formula of Roth et al. (1990). Thereby, snow is a mixture of water, ice, and air. The relative permittivity of snow $\epsilon_{\mathrm{r}, \mathrm{s}}^{\prime}$ depends on the dry snow to ice density ratio (dry snow density $\rho_{\mathrm{ds}}=370 \frac{\mathrm{kg}}{\mathrm{m}^{3}}$, ice density $\rho_{\mathrm{i}}=917 \frac{\mathrm{kg}}{\mathrm{m}^{3}}$ ), wetness $W$ (in percent per volume), and the relative permittivities of water $\epsilon_{\mathrm{r}, \mathrm{w}}^{\prime}$, ice $\epsilon_{\mathrm{r}, \mathrm{i}}^{\prime}$, and air $\epsilon_{\mathrm{r}, \mathrm{a}}^{\prime}$ :

$$
\begin{aligned}
\epsilon_{\mathrm{r}, \mathrm{s}}^{\prime}= & \left(0.01 \cdot W \sqrt{\epsilon_{\mathrm{r}, \mathrm{w}}^{\prime}}+\frac{\rho_{\mathrm{ds}}}{\rho_{\mathrm{i}}} \sqrt{\epsilon_{\mathrm{r}, \mathrm{i}}^{\prime}}\right. \\
& \left.+\left(1-\frac{\rho_{\mathrm{ds}}}{\rho_{\mathrm{i}}}-0.01 \cdot W\right) \cdot \sqrt{\epsilon_{\mathrm{r}, \mathrm{a}}^{\prime}}\right)^{2}
\end{aligned}
$$

The loss factor $\epsilon_{\mathrm{r}, \mathrm{s}}^{\prime \prime}$ in snow is calculated according to Sihvola and Tiuri (1986). It strongly depends on wetness:

$\epsilon_{\mathrm{r}, \mathrm{s}}^{\prime \prime}=\frac{f}{10^{9} \mathrm{~Hz}} \cdot \epsilon_{\mathrm{r}, \mathrm{w}}^{\prime \prime} \cdot\left(0.001 \cdot W+8.0 \cdot 10^{-5} \cdot W^{2}\right)$.

The loss factor of dry snow is approximately zero and thus negligible.

\subsection{Reflection}

The reflection occurs due to a change in the relative permittivity at the air/medium boundary. Due to the reflection, only
Table 1 Relative permittivity $\epsilon_{\mathrm{r}, \mathrm{m}}^{\prime}$ and $\epsilon_{\mathrm{r}, \mathrm{m}}^{\prime \prime}$ for different media dependent on wetness $W$ at GPS L1 wavelength

\begin{tabular}{llll}
\hline Medium & $W$ in $\%$ & $\epsilon_{\mathrm{r}, \mathrm{m}}^{\prime}$ & $\epsilon_{\mathrm{r}, \mathrm{m}}^{\prime \prime}$ \\
\hline Dry snow & 0 & 1.73 & Approx. 0 \\
Moist snow & 2 & 2.19 & 0.03 \\
Wet snow & 6 & 3.27 & 0.12 \\
Very wet snow & 12 & 5.30 & 0.32 \\
Ice at $0{ }^{\circ} \mathrm{C}$ & - & 3.18 & 0.0006 \\
Freshwater at $0{ }^{\circ} \mathrm{C}$ & 100 & 85.1 & 8.56 \\
Air & - & 1.0 & 0 \\
\hline
\end{tabular}

a part of the entire intensity reaching the medium transmits into the medium. The incident right-hand-circular-polarized GPS signal can be described using two planar waves, a horizontal and vertical wave, which are $90^{\circ}$ phase shifted (e.g. Koch et al. 2014). The linear vertical and horizontal reflection coefficients:

$$
\begin{aligned}
& r_{\mathrm{V}}=\frac{\epsilon_{\mathrm{r}, \mathrm{m}} \cos \nu_{\mathrm{a}}-\sqrt{\epsilon_{\mathrm{r}, \mathrm{m}}-\sin ^{2} v_{\mathrm{a}}}}{\epsilon_{\mathrm{r}, \mathrm{m}} \cos \nu_{\mathrm{a}}+\sqrt{\epsilon_{\mathrm{r}, \mathrm{m}}-\sin ^{2} v_{\mathrm{a}}}} \text { and } \\
& r_{\mathrm{H}}=\frac{\cos \nu_{\mathrm{a}}-\sqrt{\epsilon_{\mathrm{r}, \mathrm{m}}-\sin ^{2} v_{\mathrm{a}}}}{\cos \nu_{\mathrm{a}}+\sqrt{\epsilon_{\mathrm{r}, \mathrm{m}}-\sin ^{2} v_{\mathrm{a}}}}
\end{aligned}
$$

depend on the relative permittivity of the medium $\epsilon_{\mathrm{r}, \mathrm{m}}$ and the incident zenith angle $\nu_{\mathrm{a}}$ of the GPS signal (Woodhouse 2005). Since the GNSS signal is circularly polarized, it is convenient to express the linear reflection coefficients as circular-polarized reflection coefficients with a co- and crosspolarized component (e.g. Löfgren 2014):

$r_{\mathrm{co}}=\left|\frac{r_{\mathrm{H}}+r_{\mathrm{V}}}{2}\right|$ and $r_{\mathrm{cross}}=\left|\frac{r_{\mathrm{H}}-r_{\mathrm{V}}}{2}\right|$

The real part of the complex circular reflection coefficients can be used to describe the magnitude of the reflected signal relative to the incident signal. The mean reflectivity $R$ for an equal mix of horizontal and vertical polarizations is defined as (e.g. Koch et al. 2014):

$R=\frac{r_{\mathrm{H}}^{2}+r_{\mathrm{V}}^{2}}{2}$

Figure 2 illustrates the magnitude of the co- and crosspolarized reflection coefficients for different wetness types of snow, ice, and water for incident zenith angles from $0^{\circ}$ to $90^{\circ}$. The different media have a high influence on the crosspolarized coefficient. The higher the wetness in snow, the 


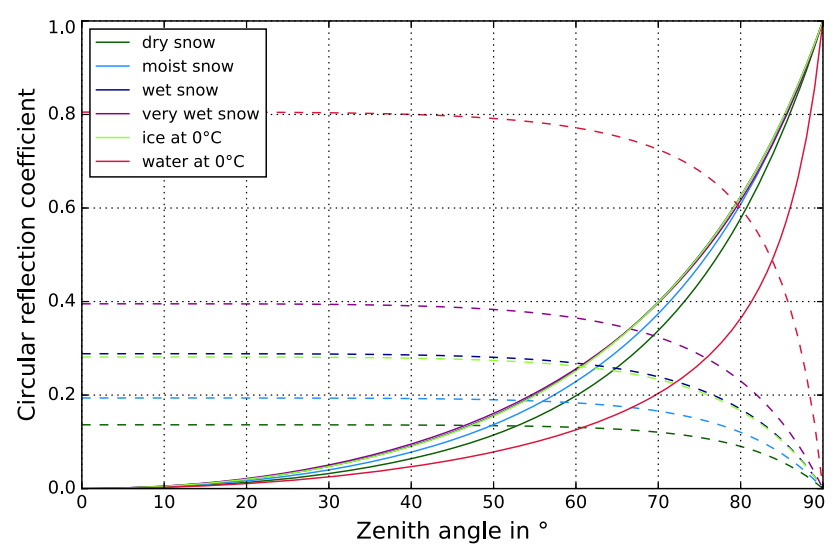

Fig. 2 Reflection at air/medium boundary. Solid lines show the magnitude (real part) of the co-, dashed lines of the cross-polarized reflection coefficients

higher the reflection. For ice, the reflection is almost similar to wet snow. The highest reflection occurs for water with $80 \%$ reflection at zenith angles below $60^{\circ}$. Generally, the cross-polarized reflection coefficient remains almost constant below $60^{\circ}$ zenith angles and decreases strongly at large zenith angles.

The co-polarized reflection coefficients decrease to zero at the zenith in an equal dimension for the different media, except for water with the strongest decrease at high zenith angles.

The intersection between the co- and cross-polar reflection $\left(r_{\mathrm{co}}=r_{\text {cross }}\right)$ defines the Brewster angle (e.g. Löfgren 2014). The Brewster angle $B$ (Table 2) is calculated based on the real part of the refractive indices of air $n_{\mathrm{a}}^{\prime}$ and the medium $n_{\mathrm{m}}^{\prime}$ :

$B=\arctan \frac{n_{\mathrm{m}}^{\prime}}{n_{\mathrm{a}}^{\prime}}$

where the complex refractive index is the square root of the complex relative permittivity $n=\sqrt{\epsilon}_{\mathrm{r}}$.

The polarization of the reflected and transmitted GPS signal changes at the Brewster angle from right-handed (RHCP) to left-handed (LHCP) circular polarization. Dedicated GPS antennas are highly sensitive to RHCP signals, whereas the gain pattern for LHCP signal reception is kept to a minimum (Moernaut and Orban 2009). Consequently, signals transmitting into the medium with the polarization changed to LHCP can hardly be tracked anymore with geodetic GPS antennas.

\subsection{Refraction}

The transmitted signal is refracted at the air/medium boundary. This decreases the angle of incidence $v_{\mathrm{m}}$ and shortens
Table 2 Brewster angle $B$, real and imaginary part of the refractive index $n_{\mathrm{m}}^{\prime}$ and $n_{\mathrm{m}}^{\prime \prime}$ for different media at GPS L1 wavelength

\begin{tabular}{llll}
\hline Medium & Brewster angle & $n_{\mathrm{m}}^{\prime}$ & $n_{\mathrm{m}}^{\prime \prime}$ \\
\hline Dry snow & $52.77^{\circ}$ & 1.32 & 0 \\
Moist snow & $55.96^{\circ}$ & 1.48 & 0.01 \\
Wet snow & $61.08^{\circ}$ & 1.81 & 0.03 \\
Very wet snow & $66.54^{\circ}$ & 2.30 & 0.07 \\
Ice at $0{ }^{\circ} \mathrm{C}$ & $60.72^{\circ}$ & 1.78 & 0 \\
Freshwater at $0{ }^{\circ} \mathrm{C}$ & $83.82^{\circ}$ & 9.24 & 0.46 \\
\hline
\end{tabular}

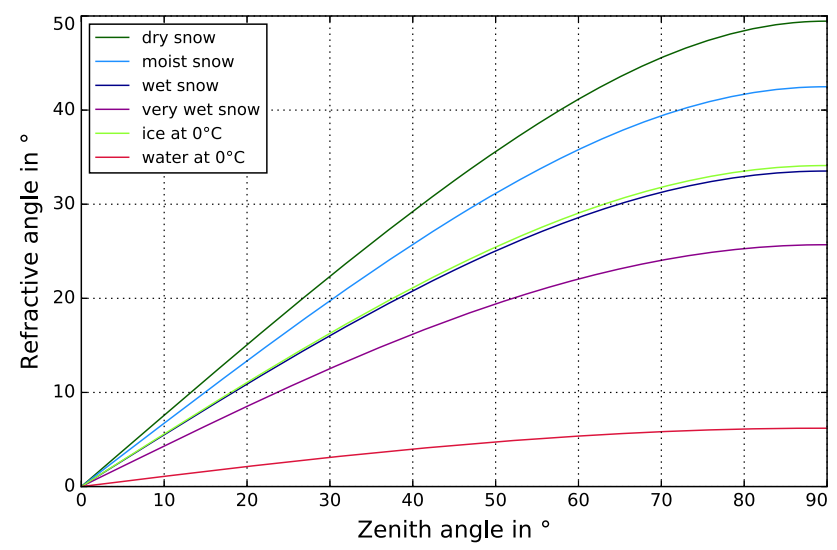

Fig. 3 Refraction at air/medium boundary

thus the geometrical distance in the medium $l_{\mathrm{m}}$ based on the lower refractive index of air $n_{\mathrm{a}}$ compared to the medium $n_{\mathrm{m}}$ (Snell-Descartes law):

$v_{\mathrm{m}}=\arcsin \frac{n_{\mathrm{a}}^{\prime} \sin v_{\mathrm{a}}}{n_{\mathrm{m}}^{\prime}}$

$l_{\mathrm{m}}=\frac{d}{\cos v_{\mathrm{m}}}$.

The signal refraction increases with wetter snow and larger incident zenith angles (Fig. 3). The refraction in ice equals almost the refraction in wet snow. Maximal refraction occurs in case of water with refractive angles below $8^{\circ}$ without a significant dependence on the zenith angle.

\subsection{Attenuation}

The power of the refracted signal in a certain distance in the medium $P\left(l_{\mathrm{m}}\right)$ is calculated based on the transmitted signal power $P_{\mathrm{t}}$ at the medium boundary. In absence of scattering, the transmitted signal power is attenuated exponentially according to the Beer-Lambert law (Ulaby and Long 2014):

$P\left(l_{\mathrm{m}}\right)=P_{\mathrm{t}} \cdot \mathrm{e}^{-\kappa_{\mathrm{m}} l_{\mathrm{m}}}$, 


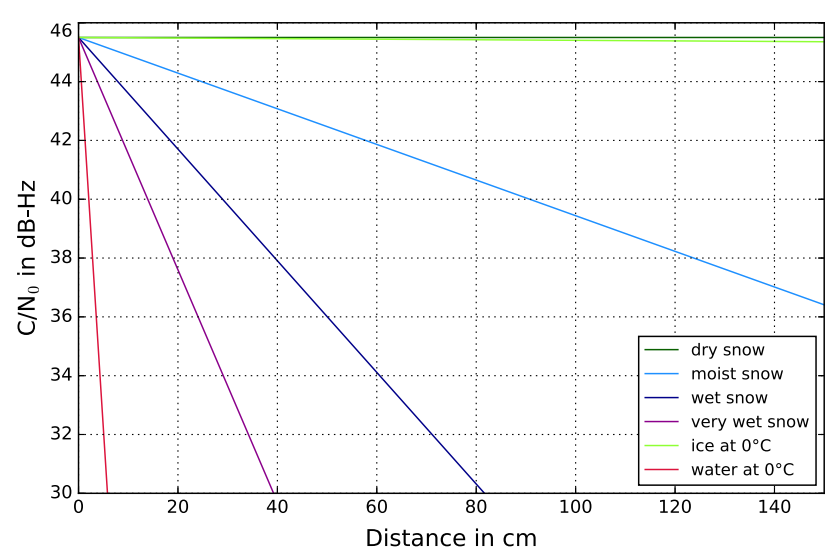

Fig. 4 Attenuation in different media for an incoming nominal signal power $C / N_{0}$ of $45.5 \mathrm{~dB}-\mathrm{Hz}$

where $\kappa_{\mathrm{m}}$ is the attenuation coefficient:

$\kappa_{\mathrm{m}}=\sqrt{\frac{\mu_{0}}{\epsilon_{\mathrm{r}, \mathrm{m}}^{\prime} \epsilon_{0}}} \cdot \epsilon_{\mathrm{r}, \mathrm{m}}^{\prime \prime} \epsilon_{0} 2 \pi f$

The attenuation encompasses energy absorption and scattering. The energy of the wave is absorbed by the electrons of the medium molecules and is transformed to an internal energy of the absorbing medium (e.g. thermal energy). Since the GPS L1 wavelength is much larger than snow particles, Rayleigh scattering occurs. The medium layer scatters isotropically as a homogeneous electric field instead of single particles (Bohren and Huffman 2007).

A measure for the incoming signal power is the carrier-tonoise density ratio $\left(C / N_{0}\right)$. The $C / N_{0}$ describes the relation of the carrier power to the noise power spectral density at the thermal noise floor in a $1 \mathrm{~Hz}$ bandwidth $\left(N_{0}=204 \mathrm{dBW}-\mathrm{Hz}\right)$. The nominal $C / N_{0}$ value is $45.5 \mathrm{~dB}-\mathrm{Hz}$ (Rao et al. 2011). The incoming signal power expressed as $C / N_{0}$ in $\mathrm{dB}-\mathrm{Hz}$ is logarithmic. Taking the logarithm of Eq. 10 leads to:

$C / N_{0}\left(l_{\mathrm{m}}\right)=C / N_{0, \mathrm{t}}-10 \kappa_{\mathrm{m}} l_{\mathrm{m}} \log _{10} \mathrm{e}$.

The transmitted signal power $C / N_{0, \mathrm{t}}$ can be expressed thereby by subtracting the reflected power from the incoming power at the medium boundary $C / N_{0}(0)$ :

$C / N_{0}\left(l_{\mathrm{m}}\right)=C / N_{0}(0)+10 \log _{10}(1-R)-10 \kappa_{\mathrm{m}} l_{\mathrm{m}} \log _{10} \mathrm{e}$.

The attenuation of the $C / N_{0}$ within a medium increases linearly with depth as well as with wetness (Fig. 4). Dry snow attenuates the GPS signal very low in contrary to water with the highest attenuation. Ice reacts very similar to dry snow. Figure 4 only shows the effect of attenuation within the different media. However, the incoming nominal signal
Table 3 Theoretical attenuation coefficient $\kappa_{\mathrm{m}}$, penetration depth $\rho_{\mathrm{m}}$, and propagation velocity $c_{\mathrm{m}}$ in different media for GPS L1 signals

\begin{tabular}{llrl}
\hline Medium & $\kappa_{\mathrm{m}}$ in $\mathrm{cm}^{-1}$ & $\rho_{\mathrm{m}}$ in $\mathrm{cm}$ & \multicolumn{1}{c}{$c_{\mathrm{m}}$ in $\frac{\mathrm{m}}{\mathrm{s}}$} \\
\hline Dry snow & 0.0 & $39^{\prime} 857.69$ & $2.28 \times 10^{8}$ \\
Moist snow & 0.007 & 143.47 & $2.02 \times 10^{8}$ \\
Wet snow & 0.022 & 45.81 & $1.66 \times 10^{8}$ \\
Very wet snow & 0.045 & 22.01 & $1.30 \times 10^{8}$ \\
Ice at $0{ }^{\circ} \mathrm{C}$ & 0.0 & $9^{\prime} 011.09$ & $1.68 \times 10^{8}$ \\
Freshwater at $0^{\circ} \mathrm{C}$ & 0.306 & 3.27 & $3.24 \times 10^{7}$ \\
\hline
\end{tabular}

power $C / N_{0}$ of $45.5 \mathrm{~dB}-\mathrm{Hz}$ is reduced by the reflectivity. The transmitted $C / N_{0, \mathrm{t}}$ decreases with an increased medium wetness.

\subsection{Signal penetration depth}

The depth at which the transmitted and attenuated signal power $P\left(l_{\mathrm{m}}\right)$ has decayed to $\frac{1}{\mathrm{e}}(37 \%)$ of its surface value, is the theoretical penetration depth of a signal propagating in a medium (Woodhouse 2005; Ulaby and Long 2014). This is also called e-folding depth with e as the Euler's number. The signal penetration depth is related to the attenuation coefficient by:

$\rho_{\mathrm{m}}=\frac{1}{\kappa_{\mathrm{m}}}$

and can be calculated in a good approximation based on the relative permittivity of the medium (Woodhouse 2005; Ulaby and Long 2014):

$\rho_{\mathrm{m}}=\frac{\lambda \sqrt{\epsilon_{\mathrm{m}}^{\prime}}}{2 \pi \epsilon_{\mathrm{m}}^{\prime \prime}}$ for $: \frac{\epsilon_{\mathrm{m}}^{\prime \prime}}{\epsilon_{\mathrm{m}}^{\prime}}<0.1$

The relationship for $\epsilon_{\mathrm{m}}^{\prime \prime} / \epsilon_{\mathrm{m}}^{\prime}$ is valid for all investigated snow types and ice. However, the relationship reaches 0.1 for freshwater.

Table 3 summarizes the calculated attenuation coefficients and penetration depths for the different snow types, ice, and water. The GPS signal penetrates dry snow and ice to a large depth (approximately 400 and $90 \mathrm{~m}$, respectively). The penetration depth reduces significantly the wetter the snow is with a limit of $3.27 \mathrm{~cm}$ for a water layer. This reduction is mainly due to the stronger signal attenuation in snow with higher wetness.

\subsection{Excess path length}

As described in Sect. 2.3, the refraction shortens the geometrical path $l_{\mathrm{m}}$ through the medium. The geometrical path 


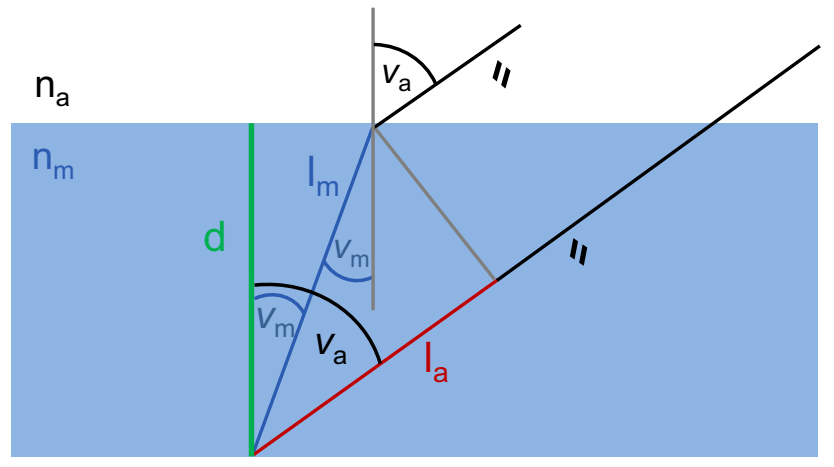

Fig. 5 Geometry of signal paths in $\operatorname{air}\left(l_{\mathrm{a}}\right)$ if no medium is present and in a medium $\left(l_{\mathrm{m}}\right)$ of depth $d$

(Fig. 5) is independent on the velocity $c_{\mathrm{m}}$ of the signal propagation in a medium. However, the propagation velocity strongly depends on the present medium based on the refractive index $n_{\mathrm{m}}$ and the vacuum speed of light $c_{0}$ (Eq. 1). The signal travel times in air $\left(t_{\mathrm{a}}\right)$ or in a present medium $\left(t_{\mathrm{m}}\right)$ are defined based on the corresponding geometrical distances $\left(l_{\mathrm{a}}, l_{\mathrm{m}}\right)$ and propagation velocities $\left(c_{0}, c_{\mathrm{m}}\right)$ :

$t_{\mathrm{a}}=\frac{l_{\mathrm{a}}}{c_{0}} ; \quad t_{\mathrm{m}}=\frac{l_{\mathrm{m}}}{c_{\mathrm{m}}}=\frac{n_{\mathrm{m}} l_{\mathrm{m}}}{c_{0}}$

$l_{\mathrm{a}}=l_{\mathrm{m}} \cos \left(v_{\mathrm{a}}-v_{\mathrm{m}}\right) ; \quad l_{\mathrm{m}}=\frac{d}{\cos v_{\mathrm{m}}}$.

The slower propagation velocity in a medium causes therefore a time delay $\delta t$ :

$\delta t=t_{\mathrm{m}}-t_{\mathrm{a}}=\frac{1}{c_{0}} \cdot\left(\frac{n_{\mathrm{m}} d}{\cos v_{\mathrm{m}}}-\frac{d \cos \left(v_{\mathrm{a}}-v_{\mathrm{m}}\right)}{\cos v_{\mathrm{m}}}\right)$.

The electrical path $L$ an electromagnetic wave propagates along in air $L_{\mathrm{a}}$ or in the presence of a medium $L_{\mathrm{m}}$, additionally depends on the corresponding travel times $\left(t_{\mathrm{a}}, t_{\mathrm{m}}\right)$ (Hofmann-Wellenhof et al. 2001):

$L_{\mathrm{a}}=c_{0} t_{\mathrm{a}} ; \quad L_{\mathrm{m}}=c_{0} t_{\mathrm{m}}$.

The time delay $\delta t$ leads to an elongation of the electrical path in the medium $L_{\mathrm{m}}$ and affects the position estimation in GPS processing. The electrical path elongation due to the presence of the medium instead of air is called the excess path length $\delta L_{\mathrm{m}}$ and depends on the refractive index $n_{\mathrm{m}}$ of the present medium and the incident angle $v_{\mathrm{a}}$ :

$$
\begin{aligned}
\delta L_{\mathrm{m}} & =L_{\mathrm{m}}-L_{\mathrm{a}}=c_{0} \cdot \delta t \\
& =d \cdot\left(\frac{n_{\mathrm{m}}}{\cos v_{\mathrm{m}}}-\frac{\cos \left(v_{\mathrm{a}}-v_{\mathrm{m}}\right)}{\cos v_{\mathrm{m}}}\right) .
\end{aligned}
$$

Introducing $v_{\mathrm{m}}$ (Eq. 8) and applying basic trigonometric transformations leads to a simplified equation for the excess path length:

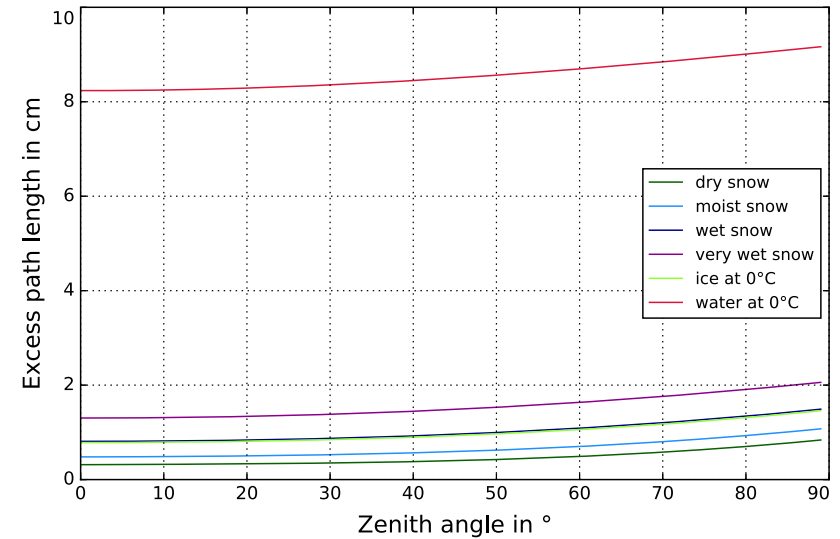

Fig. 6 Excess path length in a $1 \mathrm{~cm}$ depth layer for different media

$\delta L_{\mathrm{m}}=d \cdot\left(\sqrt{n_{\mathrm{m}}^{2}-\sin ^{2} v_{\mathrm{a}}}-\cos v_{\mathrm{a}}\right)=d \cdot F\left(v_{\mathrm{a}}\right)$

where $F\left(v_{\mathrm{a}}\right)$ is the derived mapping function, which is either used to correct the influence of the medium on the position solution or to estimate the depth $d$ of a layer (Sects. 3.6 and 3.8).

Figure 6 illustrates a strong dependence on wetness in the excess path length. Higher wetness elongates the electrical path increasingly due to a decreasing velocity $c_{\mathrm{m}}$ in the medium (Table 3 ). The electrical path in ice is similarly elongated as in wet snow. The propagation velocity in water is approximately $\frac{1}{7}$ of the velocity in dry snow and leads to a 7 times longer path. A small dependence on elevation of maximally $1 \mathrm{~cm}$ is shown for all media. This allows the water depth parameter estimation in double difference processing as it does not behave like an isotropic error, which would be canceled out.

\section{Experimental analysis of GPS L1 observations from submerged antennas}

\subsection{Basic idea}

The propagation of the GPS signal through a snowpack is highly sensitive to wetness. Increasing wetness is characterized by a higher relative permittivity, leading to more reflection at the air/snow boundary and thus less signal transmission. The transmitted signal is increasingly refracted, delayed, and attenuated while propagating through the snowpack. This results in shorter penetration depths. Ice behaves like wet snow. However, for signal attenuation, ice reacts very similar to dry snow. As shown in Sect. 2, liquid water exerts the largest influence on GPS signal propagation through a snowpack. Therefore, an experiment was set up to investigate 
Table 4 Equipment of the experimental setup (Leica Geosystems AG 2010; SensPro Electronics 2015). The same receivers and antennas are used for the submerged and the reference station

\begin{tabular}{ll}
\hline Sensor type & Properties \\
\hline Leica GR10 receiver & Multi frequency \\
& Multi GNSS \\
Leica AS10 antenna & Multi frequency \\
& Multi GNSS \\
& Gain: $27 \mathrm{dBi}$ \\
& Noise figure: $<2 \mathrm{dBi}$ \\
SensPro water level sensor & Type: SMCGO-0200MC01V013P \\
& Linearity: $0.05 \%$ \\
& Repeatability: $0.002 \%$ \\
& $1-\sigma$ accuracy: $0.1 \mathrm{~mm}$ \\
\hline
\end{tabular}

the characteristics and limitations of GPS L1 observations from an antenna submerged in water. Based on this experiment, the signal penetration depth, the receiver tracking performance, the attenuation of signal strength, and the position solutions are analyzed together with the possibility to estimate the water level above the submerged GPS antenna.

\subsection{Setup}

The experiment was set up using a pool, a water level control by communicating pipes, a geodetic GPS antenna (Leica AS10) and receiver (Leica GR10), as well as a water level sensor (SensPro SMC). The SensPro linear position sensor is based on the contactless magnetostrictive principle and was calibrated by estimating a calibration function using piled test tubes with known dimensions. Table 4 shows an overview of the sensors used in the experimental setup.

The geodetic GPS antenna was placed into the water (Fig. 7), and the water level was increased daily by a step of $2 \mathrm{~mm}$ up to $55 \mathrm{~mm}$ above the antenna surface.

A geodetic GNSS base station is set up $10 \mathrm{~m}$ next to the pool. Exactly the same equipment as for the submerged station is used (Leica AS10 antenna, Leica GR10 receiver, same antenna cable type and length) to ensure best possible consistency for all comparisons. Two additional geodetic GNSS base stations (ETH2, ETHZ with Trimble NETR9 receivers) from the Swiss permanent network are located 5-10 m outside the pool. These 2 reference stations have been used to determine best possible reference coordinates for the submerged station.

The submerged antenna was set up at $30 \mathrm{~cm}$ above the bottom of the pool to avoid reflections off the ground. GPS signals penetrate about $3.5 \mathrm{~cm}$ of freshwater (Table 3). Further propagation of a part of the signal is still possible; however, no GPS signal can penetrate $30 \mathrm{~cm}$ of water down to the bottom of the pool and then be reflected back to the

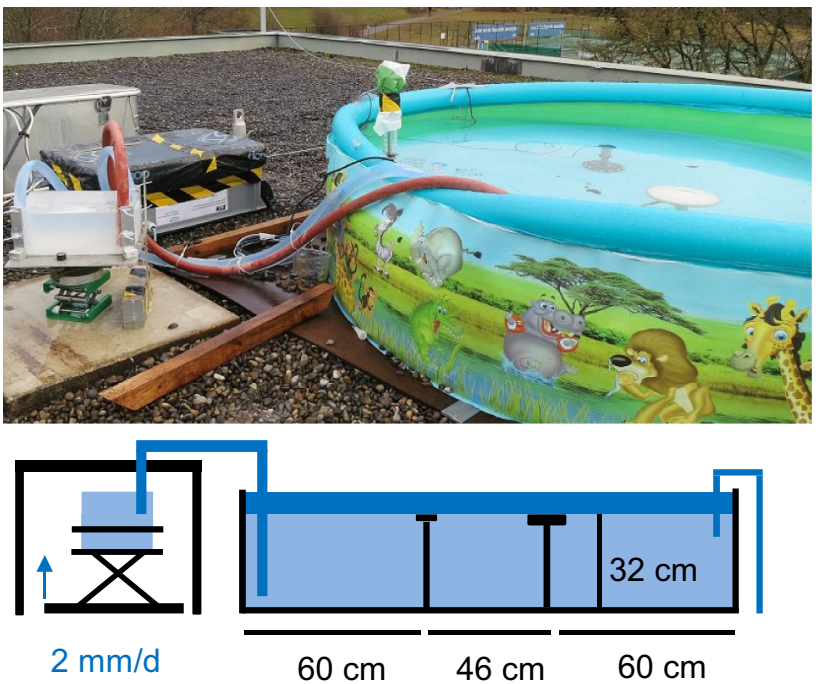

Fig. 7 Experimental setup

Table 5 Antenna field regions for the submerged geodetic Leica AS10 antenna. (Reproduced with permission from Rao et al. 2011)

\begin{tabular}{|c|c|}
\hline Field regions & Leica AS10 in cm \\
\hline Antenna diameter $D$ & 16 \\
\hline \multicolumn{2}{|l|}{ Near-fields } \\
\hline Reactive $\quad R_{1}<0.62 \sqrt{\frac{D^{3}}{\lambda}}$ & 9 \\
\hline Radiating $\quad R_{2}<\frac{2 D^{2}}{\lambda}$ & 27 \\
\hline Far-field $\quad R_{3}>\frac{2 D^{2}}{\lambda}+\lambda$ & 46 \\
\hline
\end{tabular}

antenna. Moreover, this setup respects a cutoff angle of $5^{\circ}$ to discard signals transmitting through the pool's sidewall. A low-cost antenna was also placed in the pool. However, the data of the installed low-cost system (u-blox antenna and receiver) were not yet analyzed. In a first step, the present study aims to understand and explain the effects of water on submerged antennas based on the geodetic system. The assessment of the low-cost system is planned as a followup study. The distance of $46 \mathrm{~cm}$ between the two antennas was chosen to be in each other's far-field (Table 5). This distance is chosen to mitigate possible near-fields interaction between the two antennas in the same frequency band. However, this interaction is negligible when the distance between the antennas is greater than $\frac{\lambda}{2}$ (Rao et al. 2011).

In order to keep the water level in the pool constant, a levelled reference vessel was used and connected to the main basin with three communicating pipes. A constant water inflow compensated evaporation, whereby the surplus water or impinged rain run over the border of the wind-protected reference vessel (small vessel on the left side in Fig. 7). A lifting plate was used to heighten the reference vessel. This increases the water level at the main basin. The water level was mon- 
itored by the water level sensor, which had an accuracy of $\frac{1}{10} \mathrm{~mm}$ (Table 4). Data from a permanent nearby weather station could be used to monitor rain, wind, and temperature. Two additional temperature sensors were mounted at the experimental site, one was submerged and the other one placed above the water surface.

In this setup, the antenna is directly placed into the water, whereas the receiver is sited in a box next to the pool. However, a GPS antenna is designed for an usual environment of air. The antenna impedance matches the impedance of air in order to avoid refraction effects at the antenna/air boundary. When changing the environment by placing the antennas into water, an antenna impedance mismatch could occur influencing the tracking performance (Rao et al. 2011).

\subsection{Method of investigation}

The GPS L1 data of the submerged station are processed differentially to the Leica base station data. The impact of antenna phase center offsets and variations as well as gain patterns is eliminated as the same equipment is used on both stations. Atmospheric influences are mitigated by the short baseline of approximately $10 \mathrm{~m}$. The $C / N_{0}$ of each satellite is subtracted epoch-wise from the corresponding value recorded at the base station to analyze the signal strength attenuation at $1 \mathrm{~Hz}$ sampling frequency. The ratio of the number of observations of the submerged antenna versus the number at the base station is calculated to determine the tracking performance.

Reference measurements in the empty basin are recorded for 7 days in a row at the end of the 2 months observation period (November and December 2015). The reference measurements are averaged over the 7 days and used for the analysis of the direct observations at $1 \mathrm{~Hz}$. The averaged reference measurements are further indicated as water level " -1 " in all figures. The zero water level is defined to be at the physical antenna top surface in the basin. The water level was increased from 0 to $30 \mathrm{~mm}$ by $2 \mathrm{~mm}$ steps, from 30 to $55 \mathrm{~mm}$ by $5 \mathrm{~mm}$ steps and kept constant per day.

Throughout this experiment, we assume that the interface between air and water is perfectly flat. This is, of course, an idealization as the roughness of the water surface caused by environmental influences would change signal characteristics like refraction and attenuation. To ensure that our assumption holds, days with high surface fluctuations due to stormy winds were detected and excluded based on the water level sensor and weather station data. This affected the days with water levels $4,6,14$, and $16 \mathrm{~mm}$; they were remeasured before the reference measurements. For all other days, the roughness effects are considered to be of a very small order and are assumed to average out.

All data are processed within a $17 \mathrm{~h}$ interval per day. The analysis interval is shifted each day by 4 min to ensure the same GPS satellite constellation, which repeats every $23 \mathrm{~h}$ $56 \mathrm{~min}$. In these $17 \mathrm{~h}$, no user interaction occurred with the setup over the 2 months observation period.

Precise coordinates of the submerged station are computed as a combined solution of the 7 reference days with empty basin. The position is calculated by a double difference processing with the Bernese GNSS software (Dach et al. 2015) with 30 s sampling data. The processing is done separately for all three baselines between the submerged geodetic station and the reference stations (Leica, ETH2, ETHZ). In the present paper, ambiguity fixed results are shown for the baseline between the submerged station and the ETH2 AGNES reference station. Ionospheric, clock, and precise orbit products are used for the processing of the short baseline GPS L1 data. Antenna phase center offsets and variations have been taken into account by introducing group calibrations. The external products are provided by the International GNSS Service (Dow et al. 2009).

For the days, where water was added in the pool (water level days), the positions are estimated daily and are processed similarly. The error induced by the excess path length (Sect. 2.6) when the signal propagates through a water layer above the antenna, is introduced in the zero difference phase observation equation as an additional parameter $\delta L_{\mathrm{w}}$ :

$L=\rho+\delta \rho+\delta L_{\mathrm{w}}+\lambda N+\sigma$.

Thereby, $L$ is the observed path length, $\rho$ is the range between the submerged antenna and a satellite. $\delta \rho$ includes all known path delays from the submerged antenna to the satellite (e.g., ionospheric and tropospheric delays). A detailed description of the path delays can be found in HofmannWellenhof et al. (2001). The unknown number of ambiguities is contained in $\lambda N$, and the measurement noise is expressed as $\sigma$.

The excess path length $\delta L_{\mathrm{w}}$ (Sect. 2.6) depends on the water depth $d$ above the antenna, the refractive index of water $n_{\mathrm{w}}$, and the zenith angle $v_{\mathrm{a}}$ of the observation:

$\delta L_{\mathrm{w}}=d \cdot\left(\sqrt{n_{\mathrm{w}}^{2}-\sin ^{2} v_{\mathrm{a}}}-\cos v_{\mathrm{a}}\right)=d \cdot F\left(v_{\mathrm{a}}\right)$.

$F\left(v_{\mathrm{a}}\right)$ can be interpreted as a mapping function to compute the excess path length $\delta L_{\mathrm{w}}$ for a specific zenith angle $v_{\mathrm{a}}$ from the known water depth $d$.

By forming the single difference to the base station, the satellite clock, the atmospheric and relativistic effects cancel out at short baselines. As the water only affects the submerged GPS station, the error introduced by the water remains together with the receiver clock error, ambiguities, and multi-path. The receiver clock error can be eliminated by double difference processing. The water depth parameter can be estimated, if the coordinates of the base and submerged 


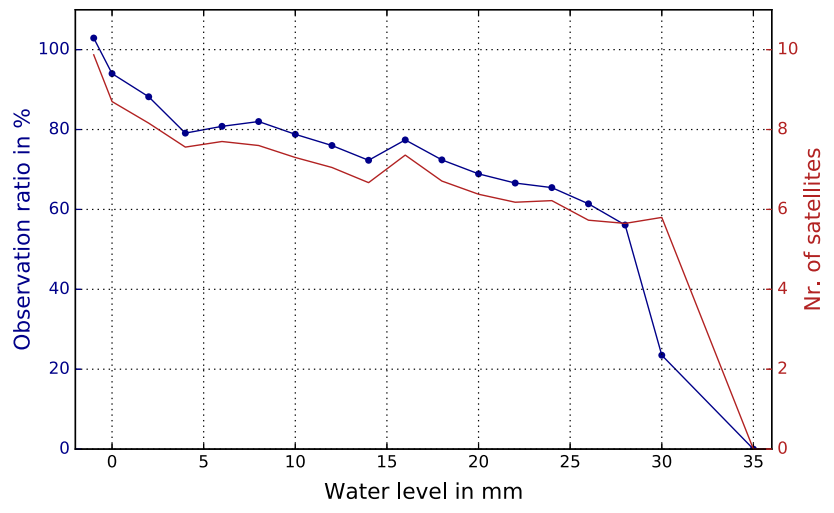

Fig. 8 GPS tracking performance in dependence of water level. The observation ratio is drawn in blue and the number of satellites in red

GPS station are precisely known and fixed. Inversely, the position solution can be corrected by applying the model, if the water depth is known.

\subsection{Tracking performance}

The tracking performance of the submerged GPS receiver is analyzed based on a comparison of the observed to the expected number of observations per water level (Rao et al. 2011). The expected number of observations could be seen during the same period without the influence of water, hence at the base station. The ratio is:

$r=\frac{N_{\mathrm{obs}}}{N_{\mathrm{exp}}}=\frac{N_{\mathrm{w}}}{N_{\mathrm{ref}}}$

Figure 8 illustrates the ratio of the observations recorded by the submerged station in relation to the base station (blue line). For the empty pool days (water level " -1 ”), the submerged Leica antenna records 5\% more observations on average as the Leica reference station. As the same equipment is used, this is due to the reference station location next to a small roof with sky obstruction in the East. The observations decrease significantly with added water in the pool. The decrease is rapid until $4 \mathrm{~mm}$, linear until $28 \mathrm{~mm}$ and abrupt afterward until $35 \mathrm{~mm}$. In consequence, the signal penetrates between 30 and $35 \mathrm{~mm}$ of fresh water and is still tracked. This corresponds to the theoretically derived signal penetration depth of $32.7 \mathrm{~mm}$ (Table 6). However, the theoretically derived value is defined as the distance where the signal power is reduced to $\frac{1}{e}$ (Sect. 2.5). Signal tracking could thus be longer possible. The tracking additionally depends on the $C / N_{0}$ tracking threshold of the receiver, which is defined by the manufacturers. The geodetic Leica GR 10 receiver uses an acquisition threshold of $30 \mathrm{~dB}-\mathrm{Hz}$ and thus effectively suppresses signals with low signal strengths. Low signal quality signals, e. g., from low elevation satellites, are thus not tracked anymore.

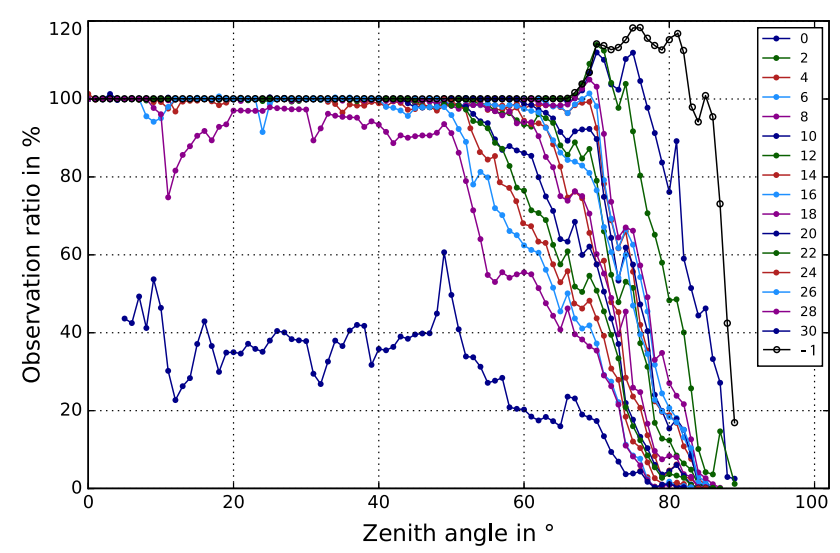

Fig. 9 GPS tracking performance in dependence of water level and zenith angle

Refraction effects reduce the $C / N_{0}$ significantly (Sect. 3.5), and signals are harder to track. The red line in Fig. 8 demonstrates the mean number of tracked satellites dependent on the water level. Less satellites are visible at higher water levels. Enough satellites are generally tracked per epoch to calculate a position solution, even though the number of observations drops significantly. The minimal value of 4 satellites is undercut after $30 \mathrm{~mm}$ for the submerged geodetic receiver.

Figure 9 shows the observation ratio according to Eq. 23 for all water levels in dependence on the zenith angle. The longer path through water at large zenith angles reduces significantly the number of observations. Furthermore, the total reflectivity is strongest at zenith angles above $85^{\circ}$, leading to minimal signal transmitting into the water. The submerged station observes as much data until $70^{\circ}$ of zenith angle for the empty pool days as the Leica reference station. The observations are thereby increased below $70^{\circ}$ zenith angle for the submerged station, which is due to a better satellite visibility because of the higher location on the small roof with less sky obstruction in the East direction. As the water level rises up to $8 \mathrm{~mm}$, the number of recorded observations is kept constant at zenith angles below $70^{\circ}$ and reduces abruptly at high zenith angles. With higher water levels, the maximal zenith angle of tracked observations decreases. No change in observations with higher water levels strikes out below $50^{\circ}$ of zenith angle, except for the highest water level of $30 \mathrm{~mm}$.

\subsection{Attenuation of signal strength}

The attenuation of the signal strength in dependence of water above the antenna surface is analyzed based on the $C / N_{0}$ values extracted directly from the observation files. In order to analyze the change in the $C / N_{0}\left(l_{\mathrm{w}}\right)$ due to the water level influence, the atmospheric effects are canceled out by build- 


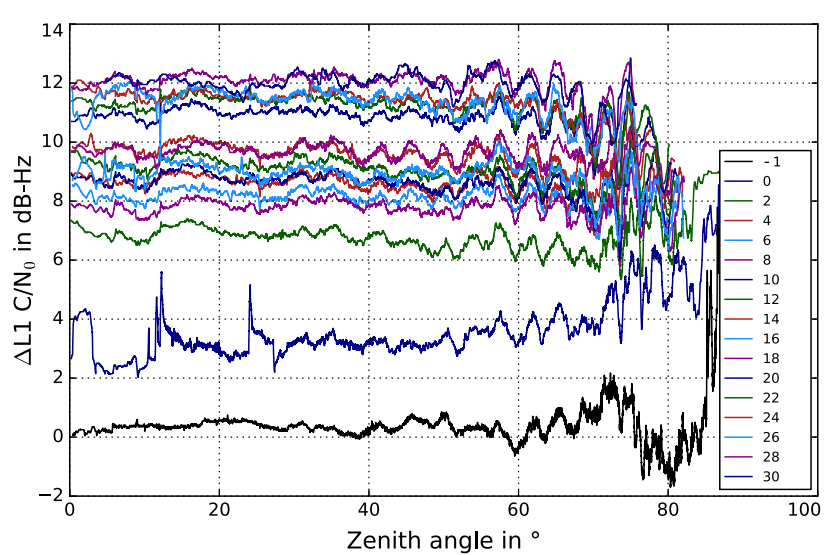

Fig. $10 \Delta C / N_{0}$ for different water levels dependent on the zenith angle. The data are median filtered for display purposes

ing a single difference to the base station:

$$
\Delta C / N_{0}\left(l_{\mathrm{w}}\right)=C / N_{0, \text { ref }}-C / N_{0}\left(l_{\mathrm{w}}\right)
$$

The $\Delta C / N_{0}\left(l_{\mathrm{w}}\right)$ as a function of the incident zenith angle is presented in Fig. 10. The differentiation increases the noise level by $\sqrt{2}$. The elevation-dependent trend from the atmospheric effects is canceled out. By reason of the high refraction in water (Fig. 3), the water layer does not introduce a new elevation-dependent trend. However, a decrease in the $\Delta C / N_{0}$ is visible for different water levels. A strong depletion is visible when the first $2 \mathrm{~mm}$ of water is added. This is caused by the high reflectivity at the water surface. More than $50 \%$ of the signal is reflected at the water surface. The transmitted signal strength is decreased dependent on the incident zenith angle of the signal with minimal transmission at zenith angles above $85^{\circ}$. A further decrease in the signal strength could be due to the change in the antenna environment from air (empty basin) to water ( 0 and $2 \mathrm{~mm}$ water level). Thereby, a strong increase in the refractive index is present from air $n_{\mathrm{a}}^{\prime}=1$ to water $n_{\mathrm{w}}^{\prime}=9.24$ (Table 2). The $C / N_{0}$ decreases considerably less above $2 \mathrm{~mm}$. Reflection stays constant with increasing water level. Thus, only attenuation is present, which decreases the signal strength less than the reflection at the water surface. Multi-path signals are present at the empty basin and at zero water level as well as for all base station observations. For higher water levels, the amplitude of the multi-path signals is weaker due to the signal attenuation. These multi-path signals influence the observations strongest above $60^{\circ}$ zenith angle. The differentiation to the base station induces therefore multi-path variations to all water levels above $60^{\circ}$ of zenith angle.

Figure 11 indicates the mean and standard deviation of the $\Delta C / N_{0}\left(l_{\mathrm{w}}\right)$ per water level. Observations from high zenith angles above $60^{\circ}$ are excluded due to the high influence of multi-path signals. This illustrates a strong depletion in the first $2 \mathrm{~mm}$ of water as well as a weaker linear decrease with

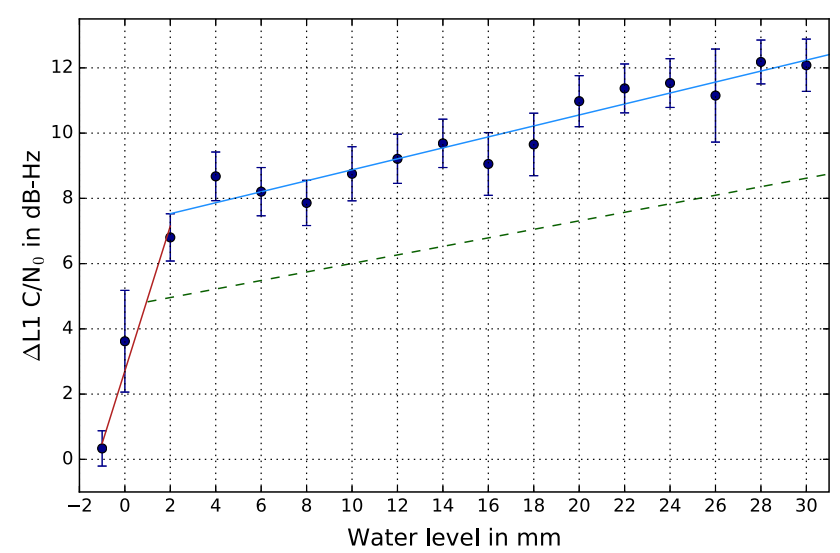

Fig. 11 Experimental attenuation of $\Delta C / N_{0}$ in dependence of the water level. The dashed line illustrates the theoretical attenuation within water for visual comparison

increasing water level. The high reflectivity of water causes the strong depletion. Calculating the reflected power leads to a loss of approximately $5 \mathrm{~dB}-\mathrm{Hz}$ (Eq. 13). The theoretical loss through reflectivity is indicated in Fig. 11 with the offset in the beginning of the theoretical attenuation curve (dashed green line). The residual loss in the strong $\Delta C / N_{0}\left(l_{\mathrm{w}}\right)$ depletion is assumed to be caused by an antenna mismatch in the water environment (Sect. 3.2). The decrease at higher water levels should be due to a longer propagation in the attenuating water layer.

The logarithmic attenuation function (Eq. 25) is fitted separately to the data for the slope from 0 to $2 \mathrm{~mm}$ and the slope above $2 \mathrm{~mm}$ water level. The attenuation is calculated according to Eq. 13. The signal has the same power level when reaching the base station and the water surface (Fig. 1). Therefore, the surface term $C / N_{0}\left(l_{\mathrm{w}}=0\right)$ is set equal to the observation at the base station $C / N_{0, \text { ref }}$. When inserting Eqs. 13 and 24 reduces to:

$\Delta C / N_{0}\left(l_{\mathrm{w}}\right)=-10 \log _{10}(1-R)+10 \kappa_{\mathrm{w}} l_{\mathrm{w}} \log _{10} e$

The attenuation coefficient in water $\kappa_{\mathrm{W}}$ is estimated based on Eq. 25 for the water levels above zero and indicated in Table 6 . The experimentally derived attenuation coefficient of $0.42 \frac{1}{\mathrm{~cm}}$ agrees with theory quite well. If the $2 \mathrm{~mm}$ water level is excluded from the estimation process, however, the estimated attenuation coefficient is $0.34 \frac{1}{\mathrm{~cm}}$ (value in parenthesis in Table 6) which is very close to the theoretical value. For the $2 \mathrm{~mm}$ water level, the antenna is only slightly covered by water and signal surface waves might still cause significant anomalous multi-path.

\subsection{Coordinates of submerged station}

Placing the antenna under water affects the position estimation. Effects are visible in the east, north, and up component 
Table 6 Comparison of the attenuation coefficient $\kappa_{\mathrm{W}}$ and penetration depth $\rho_{\mathrm{w}}$ in water at GPS L1 wavelength. The values in parenthesis are obtained if the $2 \mathrm{~mm}$ water level is excluded

\begin{tabular}{llll}
\hline Parameter & Theoretical & Experimental & RMS \\
\hline$\kappa_{\mathrm{W}}$ in $\mathrm{cm}^{-1}$ & 0.31 & $0.42(0.34)$ & $10^{-4}\left(10^{-4}\right)$ \\
$\rho_{\mathrm{w}}$ in $\mathrm{cm}$ & 3.27 & Approx. 3 & \\
\hline
\end{tabular}

Table 7 Quality of position (with applied model) and water depth $(d)$ estimation from Bernese. The difference between the estimated and real water depth is labeled as $\Delta d$, and the calculated value without the outlier water levels $(0,24 \mathrm{~mm})$ is given in parenthesis

\begin{tabular}{llllll}
\hline & \multicolumn{4}{c}{ RMS in mm } & \multicolumn{2}{c}{$\Delta d$ in mm } \\
& East & North & Up & $d$ & \\
\hline Mean & 0.10 & 0.13 & 0.12 & 0.24 & $0.42(0.00)$ \\
SD & 0.00 & 0.06 & 0.04 & 0.08 & $1.25(0.50)$ \\
\hline
\end{tabular}

The difference between the estimated and real water depth is labeled as $\Delta d$, and the calculated value without the outlier water levels $(0,24 \mathrm{~mm})$ is given in parenthesis

over the 2 months observation period. Figure 12 shows the three coordinate components as differences $(d E, d N, d U)$ with respect to the combined solution of the empty reference days. Thereby, no water was in the pool for 7 days in a row at the end of the observation period. The empty days show a sub-millimeter repeatability over the 7 days and are thus used as a stable reference. The model given by Eq. 22 was applied for all days to correct for the excess path delay caused by the water layer.

A certain degradation of the coordinate quality is expected as the signal reflection, and attenuation leads to a decreased $C / N_{0}$ (Fig. 11) and thus to an increased noise and a decreased number of observations for higher water levels. Figure 12 shows that, as soon as water is present, the coordinate scatter increases compared to the reference days. However, it does not get significantly worse with increasing water level. This is in accordance with Fig. 11, showing the strongest attenuation for the first $2 \mathrm{~mm}$ of water due to reflectivity and then a moderate attenuation for the remaining water levels.

According to Fig. 8, the number of tracked satellites decreases from 10 satellites at $0 \mathrm{~mm}$ water level to 6 at $30 \mathrm{~mm}$ water level. But in all cases, the resulting observation geometry is obviously still sufficient to provide good coordinate estimates.

The quality of the position and water depth estimation over all water levels is expressed as the root-mean-square error (RMS) and summarized in Table 7. The average coordinate RMS is very good and lies around $0.1 \mathrm{~mm}$. The small standard deviations of the average RMS errors $(\leq 0.06 \mathrm{~mm})$ confirm that the coordinate quality does not vary significantly for the different water levels.

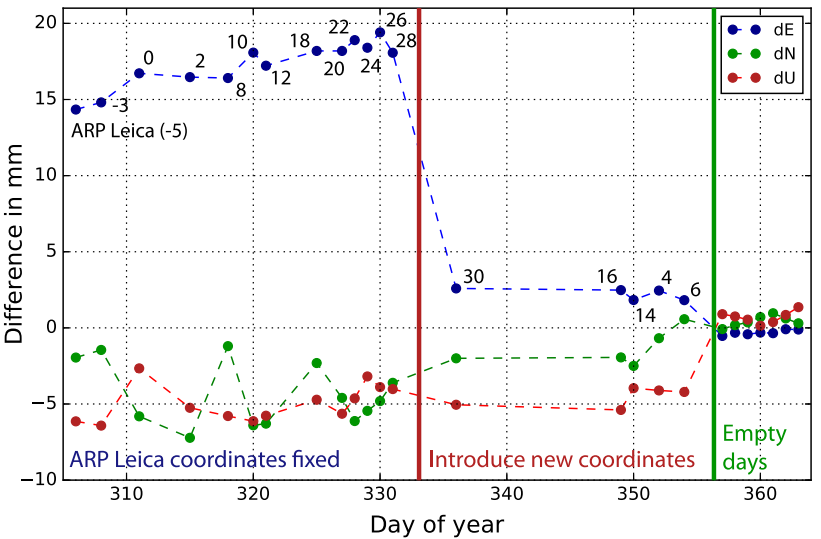

Fig. 12 Estimated position with applied model relative to the combined reference solution from the 7 empty days. Water levels are labeled in $\mathrm{mm}$ next to the east component values

An offset in the east, north, and up component is present with respect to the combined solution of the 7 stable empty reference days. The offset of $4 \mathrm{~mm}$ in average is visible at all water levels, as well as when the water level was at the ARP of the submerged geodetic antenna. This surprises, because the antenna is not yet surrounded by water at this level. This discards the assumption that the offset is caused by an impedance change due to the different antenna environment. However, it has to be influenced by the presence of water as the reference days agree to each other very well. The east component exhibits a small positive trend until $28 \mathrm{~mm}$ of water depth and a significant jump of $15 \mathrm{~mm}$ toward the water depth of $30 \mathrm{~mm}$. According to Fig. 8, there are six satellites tracked on each of the days with 28 and $30 \mathrm{~mm}$ water level. Consequently, the jump is not caused by the number of tracked satellites. As the displacement is present in each of the three processed baselines, a (unknown) physical cause is assumed.

\subsection{Influence on height component estimation}

As refraction in water is high for all incident angles, the main effect on a submerged antenna is expected in the height component due to the signal path elongation.

Figure 13 illustrates the difference in the estimated height component with respect to the water level at the antenna reference point (ARP) of the submerged antenna. A linear increase is visible for the difference in the height component (height error) when the model is not applied (blue dots). The slope $m=0.9$ of the linear fitted trend and the cross-correlation coefficient $\mathrm{cc}=0.99$ (Table 8) show a high correlation between the error in the height component and the water level above the antenna. The effect of the water depth above the antenna is thus directly mapped to the height component. Therefore, the height error itself could be used to estimate 


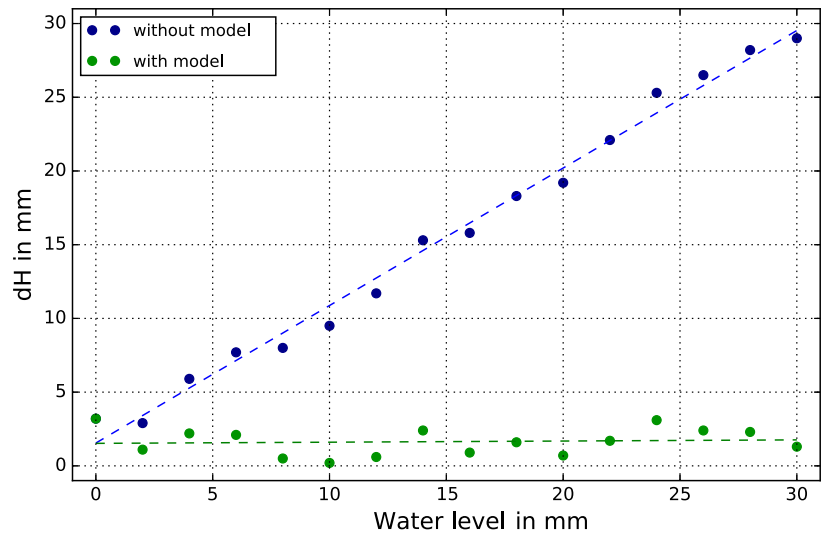

Fig. 13 Difference in height component $(d H)$ without and with model applied w.r.t. the ARP solution

Table 8 Slope $(m)$, offset $(b)$, and cross-correlation coefficient (cc) of a linear fitted trend to the difference in the height component $(\mathrm{dH})$ and the water depth $(d)$ estimation from the submerged geodetic system. The values in parenthesis are obtained when the two outlier water levels $(0$, $24 \mathrm{~mm}$ ) are removed

\begin{tabular}{llll}
\hline Parameter & $m$ & $b$ in $\mathrm{mm}$ & $\mathrm{cc}$ \\
\hline$d H$ without model & 0.9 & 1.5 & 0.99 \\
$d H$ with model & 0.0 & 1.5 & 0.08 \\
$d$ & $1.0(1.0)$ & $1.1(0.1)$ & $0.99(1.00)$ \\
\hline
\end{tabular}

the water level above the antenna. After applying the derived model (Eq. 20), the trend reduces to slope $m=0$ and the correlation is eliminated ( $\mathrm{cc}=0.08)$. The model is therefore able to correct the influence of the water depth on the height estimation, when knowing the refractive index and the depth of the present medium. However, an offset $b=1.5 \mathrm{~mm}$ (Table 8 ) is present at the linear fitted trend either by applying the model or not.

This bias results from setting the zero water level to the antenna top surface, whereas the reference height was computed with the water level at the ARP (about $3 \mathrm{~cm}$ below the antenna top surface). The offset of $1.5 \mathrm{~mm}$ is caused by the water layer between ARP and the top of the antenna and consequently reflects the arbitrary selection of references.

\subsection{Water depth estimation}

The excess path length due to the presence of a water layer of depth $d$ biases the phase observation. The water depth $d$ can be estimated if the base and submerged station coordinates as well as the refractive index of water are known. Precise a priori coordinates of the submerged station have been determined based on the combined solutions of the 7 reference days. However, the coordinates have not been stable for the

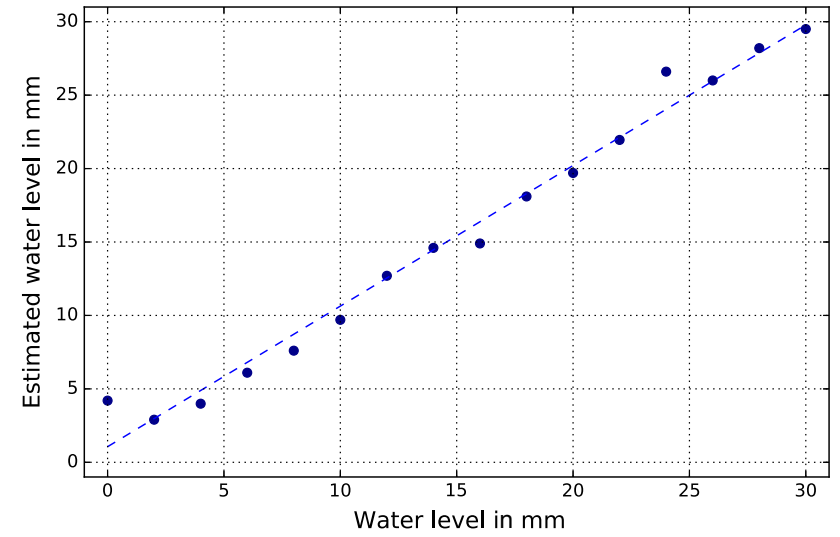

Fig. 14 Estimated water level

entire period of the experiment. A significant jump in the east component occured on doy 331 (Sect. 3.6). Therefore, the reference coordinates have been discarded for the water depth estimation. Instead, two separate sets of coordinates have been used for the days before and after the displacement. The coordinates when the water level is at the ARP of the submerged antenna are used as reference for all water levels before the jump in the east component. For the 5 days after the jump, the coordinates from the water level at $4 \mathrm{~mm}$ corrected by the water influence (by applying the model) were used as reference. The intervals with the different reference stations are marked in Fig. 12.

The mean RMS of the water depth estimation is $0.24 \mathrm{~mm}$ with a standard deviation of $0.08 \mathrm{~mm}$ as shown in Table 7 . The highest single RMS was observed at the zero water level with $0.5 \mathrm{~mm}$. The estimated water depths are on average $0.42 \mathrm{~mm}$ higher than the real water levels and scatter by $1.25 \mathrm{~mm}$. The highest difference $\Delta d=4.2 \mathrm{~mm}$ is reached at $0 \mathrm{~mm}$ water depth, followed by $\Delta d=2.6 \mathrm{~mm}$ at $24 \mathrm{~mm}$ water depth. The water level estimation at the $0 \mathrm{~mm}$ water level is outside the 3- $\sigma$ interval and thus defined as outlier. The water level at $24 \mathrm{~mm}$ is an outlier as well. Closer data inspection for that day showed a lack of stored observations for the submerged station for the first $7 \mathrm{~h}$. The reason for the outlier at the $0 \mathrm{~mm}$ water level is unknown. It might be related to anomalous multi-path caused by electromagnetic surface waves as the antenna top was exactly aligned with the water surface on that day.

After recalculating the mean and standard deviation of the water depth estimation without the two outliers at 0 and $24 \mathrm{~mm}$ water levels, the estimates fit the real values very accurately (mean of $\Delta d$ is $0 \mathrm{~mm}$ ) with a standard deviation of around $0.5 \mathrm{~mm}$ (values in parentheses in Table 7).

The high correlation of 0.99 (Table 8) between the estimated and actual present water depth can be seen in Fig. 14 . The linear fitted trend shows a slope of 1 and an offset of 
$1.1 \mathrm{~mm}$. This offset is reduced almost to zero $(0.1 \mathrm{~mm}$, cf. Table 8 ) if the two outlier water levels of 0 and $24 \mathrm{~mm}$ are removed as mentioned in the previous paragraphs. The resulting zero-offset for the water depth is now according to the expectations as the height components with and without the model both show the same offset of $1.5 \mathrm{~mm}$. The estimation of the water depth completely removes the trend from the height estimates and eliminates the correlation between height and water depth.

The water level above a submerged geodetic GPS antenna can thus be estimated precisely until the penetration depth of water is reached at $30 \mathrm{~mm}$.

\section{Conclusions}

The assumption of liquid water as the limiting parameter for GPS observations within a snowpack can be confirmed. Higher wetness in a snowpack leads to less transmission, higher refraction, higher attenuation and thus a decreased penetration depth.

A high attenuation leads also to a reduced number of observations and a loss of lock for low elevation satellites. This deteriorates the satellite geometry and thus the quality of the position solutions. Due to the high propagation delay in a freshwater layer, the height component experiences a linearly increasing error when the water level rises above the antenna. This error is highly correlated with the actual water depth and could be used directly for water depth estimation. Otherwise, the presented model enables the direct estimation of water depth when both the reference and submerged station coordinates are known.

In consequence, GPS applications within a snowpack are heavily impacted by wetness which is even more pronounced during melting periods. High wetness in a snowpack influences the tracking capability of a buried GPS antenna. On the other side, wetness in a snowpack could be estimated continuously using the attenuation of the signal strength of the buried antenna. Snow above a buried antenna biases the height component of the coordinate solution likewise to a water layer above the antenna. As the effect in the height component is dependent on the bulk relative permittivity in the snowpack, it should be possible to estimate the snow water equivalent. Furthermore, the snow water equivalent should also be determinable analogously to the water level estimate based on the presented model.

The promising results of this study encourage the assessment of the low-cost system and the comparison with the geodetic equipment in a next step.

Moreover, we want to apply our newly developed model to a seasonal snowpack in order to investigate the potential for snow water equivalent and snow depth estimation. This future investigation will be carried out using a geodetic as well as a low-cost GPS L1 system.

Acknowledgements This project is supported by the Swiss National Science Foundation (SNF 200021_156867). Special thanks to Robert Presl, technical employee of the Institute of Geodesy and Photogrammetry (IGP) at ETH Zurich, for building the system setup for the water experiment.

Open Access This article is distributed under the terms of the Creative Commons Attribution 4.0 International License (http://creativecomm ons.org/licenses/by/4.0/), which permits unrestricted use, distribution, and reproduction in any medium, provided you give appropriate credit to the original author(s) and the source, provide a link to the Creative Commons license, and indicate if changes were made.

\section{References}

Bohren C, Huffman D (2007) Absorption and scattering by an arbitrary particle, vol 10, no 1002. Wiley, New York, pp 57-81

Boniface K, Braun J, McCreight J, Nievinski F (2015) Comparison of SNODAS to GNSS reflectometry snow depth in the Western United States. Hydrolog Process. https://doi.org/10.1002/hyp. 10346

Cardellach E, Fabra F, Rius A, Pettinato S, Daddio S (2012) Characterization of dry-snow sub-structure using GNSS reflected signals. Remote Sens Environ 124:122-134

Dach R, Lutz S, Walser P, Fridez P (2015) Bernese GNSS Software version 5.2. User manual. Open Publishing

Dow J, Neilan R, Rizos C (2009) The International GNSS Service in a changing landscape of Global Navigation Satellite Systems. J Geod 83:191-198

Fierz C, Armstrong RL, Durand Y, Etchevers P, Greene E, McClung DM, Nishimura K, Satyawali PK, Sokratov SA (2009) The international classification for seasonal snow on the ground. In: IHP-VII technical documents in hydrology N 83, IACS contribution N 1

Hofmann-Wellenhof B, Lichtenegger H, Collins J (2001) Global positioning system: theory and practice. Springer, Berlin

Kaatze U (1989) Complex permittivity of water as a function of frequency and temperature. J Chem Eng Data 34:371-374

Koch F, Prasch M, Schmid L, Schweizer J, Mauser W (2014) Measuring snow liquid water content with low-cost GPS receivers. Sensors 14:20975

Koh G (1997) Complex dielectric constant of ice at $1.8 \mathrm{GHz}$. Cold Reg Sci Technol 25:119-121

Larson K, Gutmann E, Zavorotny V, Braun J, Williams M, Nievinski F (2009) Can we measure snow depth with GPS receivers? Geophys Res Lett. https://doi.org/10.1029/2009GL039430

Leica Geosystems AG (2010) Leica GR10 user manual

Löfgren J (2014) Local sea level observations using reflected GNSS signals. Ph.D. thesis, Chalmers University of Technology, Gothenburg, Sweden

Mätzler C (1998) Microwave properties of ice and snow. In: Schmitt B, De Bergh C, Festou M (eds) Solar system ices, astrophysics and space science library, vol 227. Springer, Berlin, pp 241-257

Mätzler C, Wegmüller U (1987) Dielectric properties of fresh-water ice at microwave frequencies. J Phys D Appl Phys 20(12):1623-1630. Erratum, 1988, vol 21(11):1660

Moernaut G, Orban D (2009) GNSS antennas: an introduction to bandwidth, gain pattern, polarization and all that. GPS World 20(2):42-48

Olmedo R, Olmedo A, Rico J, Sancho J, Rueda C, Perez J, Fernandez A, Ares F, Aguilera D, de Miguel P, Cerezo C (2012) Sicra: a GNSS 
cooperative system for avalanche rescue. In: 6th ESA workshop on satellite navigation technologies (Navitec 2012) and European workshop on GNSS signals and signal processing, pp 1-7

Ozeki M, Heki K (2012) GPS snow depth meter with geometry-free linear combinations of carrier phases. J Geod 86:209-219. https:// doi.org/10.1007/s00190-011-0511-x

Rao BR, Kunysz W, McDonald K (eds) (2011) GNSS antennas. Artech House, Norwood

Roth K, Schulin R, Flühler H, Attinger W (1990) Calibration of time domain reflectometry for water content measurement using a composite dielectric approach. Water Resour Res 26:2267-2273
Schleppe JB, Lachapelle G (2008) Tracking performance of a HSGPS receiver under avalanche deposited snow. GPS Solut 12:13-21

SensPro Electronics (2015) SMC: linear position sensor data sheet

Sihvola A, Tiuri M (1986) Snow fork for field determination of the density and wetness profiles of a snow pack. IEEE Trans Geosci Remote Sens 5:717-721

Ulaby FT, Long DG (2014) Microwave radar and radiometric remote sensing. Artech House Books, London

Woodhouse IH (2005) Introduction to microwave remote sensing. CRC Press, Boca Raton 